\title{
MultiLink-QuickAbw: A Multi-Congestion-Links-Oriented Fast Available Bandwidth Measurement Method
}

\author{
Junzheng Shi \\ Institute of Information Engineering, \\ Chinese Academy of Sciences, School \\ of Cyber Security, University of \\ Chinese Academy of Sciences, Beijing, \\ China, shijunzheng@iie.ac.cn, \\ Corresponding author
}

\author{
Mengyan Liu \\ Institute of Information Engineering, \\ Chinese Academy of Sciences, School \\ of Cyber Security, University of \\ Chinese Academy of Sciences, Beijing, \\ China \\ liumengyan@iie.ac.cn
}

\author{
Gang Xiong \\ Institute of Information Engineering, \\ Chinese Academy of Sciences, School \\ of Cyber Security, University of \\ Chinese Academy of Sciences, Beijing, \\ China \\ xionggang@iie.ac.cn
}

\begin{abstract}
In this research, we have implemented a fast available bandwidth measurement method for Multi-Congestion-Links in path, which is based on our previous method QuickAbw. QuickAbw is a fast available bandwidth measurement method which calculates the available bandwidth based on the link delay changes caused by probe packets. QuickAbw has the advantages of fast measurement speed and low intrusion, however it is not suitable for situations where there are multiple congested links in network path. In this study, we analyzed the link delay changes when there are multiple congested links in network path, based on the analysis of multi-link model, a universal improvement was made to QuickAbw, enabling it to measure the number of congested links and available bandwidth in the multi-links path.
\end{abstract}

\section{CCS CONCEPTS}

- Computing methodologies $\rightarrow$ Modeling and simulation; Model development and analysis.

\section{KEYWORDS}

Fast available bandwidth, measurement

\section{ACM Reference Format:}

Junzheng Shi, Mengyan Liu, and Gang Xiong. 2021. MultiLink-QuickAbw: A Multi-Congestion-Links-Oriented Fast Available Bandwidth Measurement Method. In 2021 2nd International Conference on Artificial Intelligence and Information Systems (ICAIIS '21), May 28-30, 2021, Chongqing, China. ACM, New York, NY, USA, 9 pages. https://doi.org/10.1145/3469213.3470225

\section{INTRODUCTION}

Available bandwidth is a key indicator for measuring network service quality. Applications that rely on network service quality, such as streaming media and VOIP, use the available link bandwidth to adjust the audio and video stream bit rate to bring better user experience. In addition to the measurement accuracy of the available

Permission to make digital or hard copies of all or part of this work for personal or classroom use is granted without fee provided that copies are not made or distributed for profit or commercial advantage and that copies bear this notice and the full citation on the first page. Copyrights for components of this work owned by others than ACM must be honored. Abstracting with credit is permitted. To copy otherwise, or republish, to post on servers or to redistribute to lists, requires prior specific permission and/or a fee. Request permissions from permissions@acm.org.

ICAIIS '21, May 28-30, 2021, Chongqing, China

(C) 2021 Association for Computing Machinery.

ACM ISBN 978-1-4503-9020-0/21/05 . .\$15.00

https://doi.org/10.1145/3469213.3470225 bandwidth, these applications also have high requirements for measurement speed. Faster measurement speed brings faster functional response. In addition, reducing the intrusion of the user network during the measurement process also has important practical significance, such as Reduce the user's mobile data consumption. In our previous work, we implemented QuickAbw[1], a fast available bandwidth measurement method for a single bottleneck link. This method makes measurements by sending only a small number of packets, which uses the relationship between the delay growth rate of the packets and the sending rate to calculate the bottleneck link bandwidth, however, this method does not support the measurement of the available bandwidth of multiple bottleneck links. In a complex network environment, there are multiple bottleneck links the path at the same time. For example, when two mobile users use VOIP in P2P mode, each user to the mobile base station is a bottleneck link. Based on the analysis of this study, measurement result of Quickabw in this case would be higher than the actual value.

In this study, a model of multiple bottleneck links was established. We proposed a method for identifying the number of congested links based on the model, and further proposed MultiLinkQuickAbw, a fast available bandwidth measurement method for Multi-Congestion-Links in the path.

The contents of this study are as follows: In section II we propose MultiLink-QuickAbw, a fast available bandwidth measurement method suitable for Multi-Congestion-Link in network path. In section III we verify our method through experiment, and analyze the factors leading to measurement error. The fourth section is related work, introducing the research progress of available bandwidth measurement.

\section{IMPLEMENTATION OF MULTILINK-QUICKABW}

In this section,we first introduced the relevant definitions and QuickAbw formula of our previous work. Then through mathematical modeling, it is concluded that if QuickAbw is directly used for multi-tight link measurement, the result will be higher than the actual available bandwidth value. In section $\mathrm{B}$, we propose an algorithm to identify the number of tight links, and based on this algorithm, iterative-based MultiLink-QuickAbw is proposed. When MultiLink-QuickAbw finds that there are multiple tight links in the link, it reduces the sending rate in an iterative manner until the available bandwidth can be directly calculated using the QuickAbw formula. 


\subsection{Definitions}

Path $P_{a, b}$ connecting node a and node $\mathrm{b}$ is composed of multiple links $\mathrm{L}_{1}, \mathrm{~L}_{2} \ldots \mathrm{L}_{\mathrm{n}}$. Our purpose is to measure the path-available bandwidth between node a and node $b$. Node a is called the starting point of path $P_{a, b}$ and node $\mathrm{b}$ is called the endpoint. The link bandwidth is the highest data transmission rates the link can achieve which is expressed as $C_{i}$. The bottleneck bandwidth is the smallest-link bandwidth of the path which represents the maximum transmission rate of the path $P_{a, b}$. The bottleneck bandwidth is constant, unaffected by the traffic in the link, and it can be expressed as $C_{a, b}=\min \left(C_{i}\right),(i=0,1 \ldots n)$. The background traffic is expressed as $s^{m}$.The probing traffic is formed by actively sending probing packet sequence to obtain the available bandwidth and the bottleneck bandwidth, which is expressed as $s^{R}$.We assume the rate of the probing traffic in the starting point is expressed as $R_{0}$. The receiving rate of the probing traffic in the link $\mathrm{L}_{i}$ is expressed as $\mathrm{Rs}_{i}$ and the sending rate is expressed as $\mathrm{Rr}_{i}$. The sending rate of the link background traffic is expressed as $\mathrm{Ms}_{i}$ and the receiving rate is expressed as $\operatorname{Mr}_{i}(i=0,1 \ldots n)$.

The link-available bandwidth is the remaining bandwidth in the link except for the background traffic, is expressed as $\mathrm{A}_{i}$.The pathavailable bandwidth, the smallest available bandwidth among all links in the path, is expressed as $A_{a, b}=\min \left(A_{i}\right),(i=0,1 \ldots n)$.

The tight link is the link with the smallest available bandwidth. We define that the sending rate of the total traffic in the link is Qs $i$ and the receiving rate is $\mathrm{Qr}_{i}$. We also know that $\mathrm{Qs}_{i}=\mathrm{Ms}_{i}+\mathrm{Rs}_{i}$, $\mathrm{Qr}_{i}=\mathrm{Mr}_{i}+\mathrm{Rr}_{i}$.We assume that the nodes at both ends of the link $\mathrm{L}_{i}$ are $\mathrm{N}_{i-1}$ and $\mathrm{N}_{i}$, of which the buffer size is $\mathrm{B}_{i-1}$ and $\mathrm{B}_{i}$. The congestion link is the link of which the total traffic is greater than its bandwidth, and the congestion path is the path in which the congestion link exists.

We define $\mathrm{K}_{i}$ is the delay growth rate of link $\mathrm{L}_{i}$. The definition of the delay growth rate is in our previous work [1].When there is only one congestion link, $\mathrm{K}=\mathrm{K}_{i}^{c}$. If the two different nonzero delay increase rates of the path are $\mathrm{K}^{\prime \prime}$ and $\mathrm{K}^{\prime}$, and the corresponding rates of the probing traffic in the starting point of the path are $\mathrm{R}^{\prime \prime}{ }_{0}$ and $\mathrm{R}_{0}^{\prime}$, the path-available bandwidth $\mathrm{A}$ can be expressed as QuickAbw formula:

$$
\mathrm{A}=\frac{\mathrm{K}^{\prime \prime} \mathrm{R}_{0}^{\prime}-\mathrm{K}^{\prime} \mathrm{R}_{0}^{\prime \prime}}{\mathrm{K}^{\prime \prime}-\mathrm{K}^{\prime}}
$$

The capacity of the link is

$$
\mathrm{C}_{i}=\frac{\mathrm{R}_{0}^{\prime \prime}-\mathrm{R}_{0}^{\prime}}{\mathrm{K}_{i}^{\prime \prime}-\mathrm{K}_{i}^{\prime}}
$$

\subsection{Qualitative analysis of Measurement error of QuickAbw under Multiple Congestion Links in Path}

1) Relation Analysis between Path Delay Increase Rate and Rate of Probing TrafficDuring the measurement period, the background traffic in the network is presumed to be constant and the path delay increase rate $K$ is only related to $R_{0}$. K can be expressed as a function related to $R_{0} \cdot K=\mathrm{f}\left(R_{0}\right)$.

Theorem 1 When there is a specific congestion link sequence $L_{1}^{c}, L_{2}^{c} \ldots L_{u}^{c}$, the probing traffic in link $L_{\mathrm{i}}^{c}$ is $s_{\mathrm{i}}^{c} R$ and the background traffic is $s_{\mathrm{i}}^{c}$. The receiving rate of the probing traffic and the background traffic is $m r\left(s_{\mathrm{i}}^{c} R\right)$ and $m r\left(s_{i}^{c} m\right)$, which can be expressed as a function $f_{m r\left(s_{\mathrm{i}}^{c R}\right)}\left(R_{0}\right)$ and $f_{m r\left(s_{i}^{c m}\right)}\left(R_{0}\right)$ aboutR $\mathrm{R}_{0}$. When $R_{0}{ }^{\prime \prime}>R_{0}{ }^{\prime}, f_{m r\left(s_{\mathrm{i}}^{c R}\right)}\left(R_{0}{ }^{\prime \prime}\right)>f_{m r\left(s_{\mathrm{i}}^{c R}\right)}\left(R_{0}{ }^{\prime}\right)$ and $f_{m r\left(s_{i}^{c m}\right)}\left(R_{0}{ }^{\prime \prime}\right)<$ $f_{m r\left(s_{i}^{c m}\right)}\left(R_{0}{ }^{\prime}\right)$.

Proof. When $i=1, L_{1}^{c}$ is congested firstly. The rate $m_{1}^{c}\left(s_{1}^{c}\right)$ of the sending end is constant. According to formulas (6) and (7), $f_{m r\left(s_{1}^{c R}\right)}\left(R_{0}{ }^{\prime \prime}\right)>f_{m r\left(s_{1}^{c R}\right)}\left(R_{0}{ }^{\prime}\right), f_{m r\left(s_{i}^{c m}\right)}\left(R_{0}{ }^{\prime \prime}\right)<f_{m r\left(s_{i}^{c m}\right)}\left(R_{0}{ }^{\prime}\right)$.

When $i \neq 1$, we assume that $f_{m r\left(s_{i}^{c R}\right)}\left(R_{0}{ }^{\prime \prime}\right)>$ $f_{m r\left(s_{\mathrm{i}}^{c R}\right)}\left(R_{0}{ }^{\prime}\right), f_{m r\left(s_{i}^{c m}\right)}\left(R_{0}{ }^{\prime \prime}\right) \quad<\quad f_{m r\left(s_{i}^{c m}\right)}\left(R_{0}{ }^{\prime}\right)$. According to the steady flow model assumption, for arbitrary $\mathrm{R}_{0}, f_{m r\left(s_{i}^{\text {coutput }}\right)}\left(R_{0}\right)=\gamma f_{m r\left(s_{i}^{c m}\right)}\left(R_{0}\right), 0 \leq \gamma \leq 1, \gamma$ is constant. Therefore, $f_{m r\left(s_{\mathrm{i}}^{\text {coutput }}\right)}\left(R_{0}{ }^{\prime \prime}\right)<f_{m r\left(s_{\mathrm{i}}^{\text {coutput }}\right)}\left(R_{0}{ }^{\prime}\right)$.

Case 1. $L_{\mathrm{i}}^{c+1}=L_{\substack{\mathrm{i}+1 \\ \prime \prime}}^{c}$.

$f_{m s\left(s_{i+1}^{c}{ }^{\text {cross }}\right)}\left(R_{0}^{\prime \prime}\right)+f_{m s\left(s_{i+1}^{c} \text { input }\right)}\left(R_{0}^{\prime \prime}\right)<f_{m s\left(s_{i+1}^{c}{ }^{\text {cross }}\right)}\left(R_{0}{ }^{\prime}\right)+$ $f_{m s\left(s_{i+1}^{c} \text { input }\right)}\left(R_{0}{ }^{\prime}\right)$. However, $m s\left(s_{\mathrm{i}+1}^{c}\right.$ inter $)$ and $m s\left(s_{\mathrm{i}+1}^{c}\right.$ intra $)$, keeping constant, are unrelated to $R_{0}$. Therefore, when is $L_{\mathrm{i}+1}^{c}$ congested, $f_{m s\left(s_{i+1}^{c}{ }^{m}\right)}\left(R_{0}{ }^{\prime \prime}\right)<f_{m s\left(s_{i+1}^{c}{ }^{m}\right)}\left(R_{0}{ }^{\prime}\right)$.

According to formulas (6) and (7) and the assumption of the link up one level, $f_{m r\left(s_{i+1}^{c} R^{\prime}\right)}\left(R_{0}{ }^{\prime \prime}\right)>f_{m r\left(s_{i+1}^{c} R^{R}\right)}\left(R_{0}{ }^{\prime}\right), f_{m r\left(s_{i+1}^{c} m^{m}\right)}\left(R_{0}{ }^{\prime \prime}\right)<$ $f_{m r\left(s_{i+1}^{c}{ }^{m}\right)}\left(R_{0}{ }^{\prime}\right)$.

Case 2. The higher level link of $L_{\mathrm{i}+1}^{c}$ is not $L_{\mathrm{i}}^{c}$, i.e., $L_{\mathrm{i}}^{c+1} \neq L_{\mathrm{i}+1}^{c}$. The part of the traffic $s_{\mathrm{i}}^{c}$ output sent to link $L_{\mathrm{i}+1}^{c}$ is $s^{\prime}$, and the rate of traffic $s^{\prime}$ in the sending end of $L_{\mathrm{i}+1}^{c}$ is $m s\left(s^{\prime}\right)$. At the moment,

$$
m s(s \prime)=\delta m r\left(s_{i}^{\text {couttput }}\right), 0 \leq \delta \leq 1
$$

where $\delta$ is constant. It is easy to see that $f_{m s\left(s^{\prime}\right)}\left(R_{0}{ }^{\prime \prime}\right) \leq f_{m s\left(s^{\prime}\right)}\left(R_{0}{ }^{\prime \prime}\right)$. When they are equal, $\delta$ is zero.

At the moment, the rest of the data traffic in the traffic $s_{i+1}^{c} m$ is unrelated to $\mathrm{R}_{0}$, except $s^{\prime}$. Therefore, $f_{m s\left(s_{i+1}^{c}{ }^{m}\right)}\left(R_{0}{ }^{\prime \prime}\right) \leq f_{m s\left(s_{i+1}^{c}{ }^{m}\right)}\left(R_{0}{ }^{\prime}\right)$. According to formula (6), $\left.\left.f_{m r\left(s_{i+1}^{c} m\right.}\right)\left(R_{0}^{\prime \prime}\right)<f_{m r\left(s_{i+1}^{c} m\right.}{ }^{\prime}\right)\left(R_{0}{ }^{\prime}\right)$. In conclusion, Theorem 1 is proved.

Theorem $2 f\left(R_{0}\right)$ is a continuous function of $R_{0} \in[0,+\infty)$. Proof. First, we prove the continuity of specific congestion link $f\left(R_{0}\right)$. When $R_{0} \in\left[r_{1}, r_{2}\right]$, we can get the congestion links $L_{1}^{c}, L_{2}^{c} \ldots L_{u}^{c}$ and $f\left(R_{0}\right)=\sum_{i=1}^{u} g_{i}^{c}\left(R_{0}\right)$. Therefore, we only need to prove that for any $\mathrm{i}, \mathrm{g}_{i}^{c}\left(R_{0}\right)$ is continuous on $\left[r_{1}, r_{2}\right]$. Further, we can prove for any $\mathrm{f}_{i}^{c}\left(R_{0}\right)$, it is continuous on $\left[r_{1}, r_{2}\right]$.

For any link $L_{i}^{c}, \mathrm{f}_{i}^{c}\left(R_{0}\right)$ can be expressed as

$$
f_{i}^{c}\left(R_{0}\right)=\frac{f_{m s\left(s_{\mathrm{i}}^{c R}\right)}\left(R_{0}\right)+f_{m s\left(s_{\mathrm{i}}^{c m}\right)}\left(R_{0}\right)-C_{i}}{C_{i}}
$$

We use the induction method to prove it.

Since $L_{1}^{c}, m s\left(s_{1}^{c m}\right)$ and $\mathrm{R}_{0}$ are independent and $f_{m s\left(s_{1}^{c R}\right)}\left(R_{0}\right)=R_{0}$ is continuous on $\left[r_{1}, r_{2}\right]$, we can get the following equation:

$$
f_{1}^{c}\left(R_{0}\right)=\frac{R_{0}+m s\left(s_{1}^{c m}\right)-C_{1}^{c}}{C_{1}^{c}}
$$

At this time, $f_{1}^{c}\left(R_{0}\right)$ is monotonously increased about $\mathrm{R}_{0}$ and continuous on $\left[r_{1}, r_{2}\right]$. 
According to formula (8), we can get the two following equations:

$$
\begin{gathered}
f_{m r\left(s_{i}^{c m}\right)}\left(R_{0}\right)=\frac{f_{m s\left(s_{i}^{c m}\right)}\left(R_{0}\right)}{f_{m s\left(s_{i}^{c R}\right)}\left(R_{0}\right)+f_{m s\left(s_{i}^{c m}\right)}\left(R_{0}\right)} C_{i}^{c} \\
f_{m r\left(s_{i}^{c R}\right)}\left(R_{0}\right)=\frac{f_{m s\left(s_{i}^{c R}\right)}\left(R_{0}\right)}{f_{m s\left(s_{i}^{c R}\right)}\left(R_{0}\right)+f_{m s\left(s_{i}^{c m}\right)}\left(R_{0}\right)} C_{i}^{c}
\end{gathered}
$$

For link $L_{i}^{c}$, if $f_{m s\left(s_{i}^{c R}\right)}\left(R_{0}\right)$ and $f_{m s\left(s_{i}^{c m}\right)}\left(R_{0}\right)$ are continuous, $f_{m r\left(s_{i}^{c m}\right)}\left(R_{0}\right)$ and $f_{m r\left(s_{i}^{c R}\right)}\left(R_{0}\right)$ are also continuous. According to formula (8), $f_{m s\left(s_{i+1}^{c} R\right)}\left(R_{0}\right)=f_{m r\left(s_{i}^{c R}\right)}\left(R_{0}\right)$. Therefore, $f_{m s\left(s_{i+1}^{c}{ }^{R}\right)}\left(R_{0}\right)$ are continuous on $\left[r_{1}, r_{2}\right]$.

In $s_{i+1}^{c} m$, we can set $\mathrm{s}^{\prime}$ as the probing traffic that passes through $L_{i}^{c}$, and $\mathrm{s}^{\prime \prime}$ as the probing traffic that does not pass through $L_{i}^{c}$. There is $\operatorname{ms}\left(s_{i+1}^{c} m^{m}\right)=\mathrm{ms}\left(\mathrm{s}^{\prime}\right)+\mathrm{ms}\left(\mathrm{s}^{\prime \prime}\right)$. Because $\mathrm{ms}\left(\mathrm{s}^{\prime \prime}\right)$ and $\mathrm{R}_{0}$ are independent, $\mathrm{ms}\left(\mathrm{s}^{\prime \prime}\right)$ can expressed as

$$
f_{\mathrm{ms}\left(\mathrm{s}^{\prime}\right)}\left(R_{0}\right)=\rho f_{m r\left(s_{i}^{\text {coutput }}\right)}\left(R_{0}\right), 0 \leq \rho \leq 1
$$

According to the constant traffic hypothesis,

$$
f_{m r\left(s_{i}^{\text {coutput }}\right)}\left(R_{0}\right)=\varphi f_{m r\left(s_{i}^{c m}\right)}\left(R_{0}\right), 0 \leq \varphi \leq 1
$$

When $f_{m r\left(s_{i}^{c m}\right)}\left(R_{0}\right)$ is a continuous function, $f_{m r\left(s_{i}^{\text {coutput }}\right)}\left(R_{0}\right)$ is also a continuous function. Therefore, $f_{i+1}^{c}\left(R_{0}\right)$ is a continuous function.

At this time, we come to the conclusion that when there is a specific congestion link, $f\left(R_{0}\right)$ is a continuous function. In conclusion, Theorem 1 is proved.

Theorem $3 f\left(R_{0}\right)$ has a linear relation with $\mathrm{R}_{0}$ when congestion links in a particular sequence exist in the path.

Proof. We assume that congestion links in a particular sequence that exist in the path are $L_{1}^{c}, L_{2}^{c} \ldots L_{u}^{c}$.

$$
f\left(R_{0}\right)=\frac{T+\Delta d}{T}-1=\frac{m s\left(s_{1}^{c R}\right)}{m r\left(s_{\mathrm{u}}^{c R}\right)}-1 .
$$

We need only to prove that $\frac{m s\left(s_{1}^{c R}\right)}{m r\left(s_{\mathrm{u}}^{c R}\right)}$ has a linear relation with $\mathrm{R}_{0}$. For the first congestion link, $\frac{m s\left(s_{1}^{c R}\right)}{m r\left(s_{1}^{c R}\right)}=\frac{R_{0}}{c}+\frac{m s\left(s_{i}^{c m}\right)}{\mathrm{c}}, m s\left(s_{i}^{c m}\right)$ has nothing to do with $\mathrm{R}_{0}$, and $\frac{m s\left(s_{1}^{c R}\right)}{m r\left(s_{1}^{c R}\right)}$ has a linear relation with $\mathrm{R}_{0}$. If $\frac{m s\left(s_{1}^{c R}\right)}{m r\left(s_{\mathrm{i}}^{c R}\right)}$ has a linear relation with $\mathrm{R}_{0}$, we assume that $\frac{m s\left(s_{1}^{c R}\right)}{m r\left(s_{\mathrm{i}}^{c R}\right)}=$ $\mathrm{a} R_{0}+b$. Therefore,

$$
m r\left(s_{\mathrm{i}+1}^{c}{ }^{R}\right)=\frac{\frac{C_{\mathrm{i}}^{c} m s\left(s_{\mathrm{i}}^{c R}\right)}{m s\left(s_{\mathrm{i}}^{c R}\right)+m s\left(s_{\mathrm{i}}^{c m}\right)}}{\left.\frac{m s\left(s_{\mathrm{i}}^{c R}\right) * C_{\mathrm{i}}^{c}}{m s\left(s_{\mathrm{i}}^{c R}\right)+m s\left(s_{\mathrm{i}}^{c m}\right)}+\frac{\gamma m s\left(s_{\mathrm{i}}^{c m}\right) * C_{\mathrm{i}}^{c}}{m s\left(s_{\mathrm{i}}^{c R}\right)+m s\left(s_{\mathrm{i}}^{c m}\right)}+m s\left(s_{i}^{\text {intra }}\right)+m s\left(s_{i}^{\text {inter }}\right)\right)}
$$

$C_{\mathrm{i}+1}^{c} \gamma$ is the percentage of $s_{\mathrm{i}}^{c} m$ in the background traffic sent from $L_{\mathrm{i}}^{c} m$ to $L_{\mathrm{i}+1}^{c} m$. Now,

$$
\frac{m s\left(s_{1}^{c R}\right)}{m r\left(s_{i+1}^{c} R^{R}\right)}=\frac{R_{0}}{C_{i+1}^{c}}+\left(\frac{m s\left(s_{i}^{c m}\right)\left(m s\left(s_{i}^{\text {intra }}\right)+m s\left(s_{i}^{\text {inter }}\right)\right)}{C_{i+1}^{c} C_{i}^{c}}+\frac{\gamma m s\left(s_{i}^{c m}\right)}{C_{i+1}^{c}}\right)
$$$$
\left(a R_{0}+b\right)+\frac{m s\left(s_{i}^{\text {intra }}\right)+m s\left(s_{i}^{\text {inter }}\right)}{C_{i+1}^{c} C_{i}^{c}}
$$

$\frac{m s\left(s_{1}^{c R}\right)}{m r\left(s_{\mathrm{i}+1}^{c} R\right)}$ has a linear relation with $\mathrm{R}_{0}$, therefore, $\frac{m s\left(s_{1}^{c R}\right)}{m r\left(s_{\mathrm{u}}^{c R}\right)}$ has a linear relation with $\mathrm{R}_{0}$.
In conclusion, Theorem 3 is proved.

Theorem $4 f\left(R_{0}\right)$ generates congestion sequences $L_{1}^{c}, L_{2}^{c} \ldots L_{u}^{c}$ in the domain of $\left[r_{1}, r_{2}\right]$. If we add one congestion link to the sequences, $f\left(R_{0}\right)$ will get bigger on the condition that $\mathrm{R}_{0}$ remains unchanged. Proof. We assume that the congestion link at the last is $L_{u^{\prime}}^{c}$ after adding a congestion link $L_{i}^{c}$ and $f\left(R_{0}\right)^{\prime}=\frac{m s\left(s_{1}^{c R}\right)}{m r\left(s_{\mathrm{u}^{\prime}}^{c R}\right)}-1$. If $L_{i}^{c}$ is the last congestion link, it is obvious that $f\left(R_{0}\right)^{\prime}>f\left(R_{0}\right)$. If $L_{i}^{c}$ is added to other locations, it is easy to prove that $m r\left(s_{\mathrm{u}}^{c R}\right)>m r\left(s_{\mathrm{u}^{\prime}}^{c R}\right)$ through the inductive method. At the moment, $f\left(R_{0}\right)^{\prime}>f\left(R_{0}\right)$. In conclusion, Theorem 4 is proved.

2) Measuring Error AnalysisWe assume that the rates of the probing traffic are $\mathrm{R}_{0}{ }_{0}$ and $\mathrm{R}_{0}{ }_{0}\left(\mathrm{R}_{0}{ }_{0}>\mathrm{R}_{0}{ }_{0}\right)$, and the corresponding path delay increase rates actually measured are $\mathrm{K}$ and $\mathrm{K}$. The available bandwidth is calculated to be $\mathrm{A}^{m}$. The delay increase rates measured in the tight link on both sides according to $\mathrm{R}_{0}^{\prime \prime}$ and $\mathrm{R}_{0}^{\prime}$ are $\mathrm{K}^{e^{\prime \prime}}$ and $\mathrm{K}^{e \prime}$, and the actual available bandwidth is supposed to be $\mathrm{A}^{e}$.

$\mathrm{K}^{e^{\prime \prime}}=\frac{\left(\mathrm{R}^{\prime \prime}{ }_{0}-\mathrm{A}\right)}{C_{i}}, \mathrm{~K}^{e \prime}=\frac{\left(\mathrm{R}_{0}^{\prime}-\mathrm{A}\right)}{C_{i}}$. Therefore, the error $\mathrm{d}$ can be expressed as

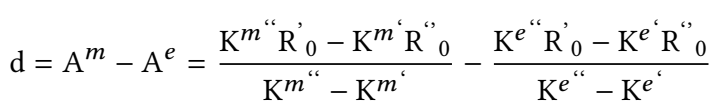

We suppose that $\eta\left(\mathrm{R}_{0}^{\prime \prime}, \mathrm{R}_{0}^{\prime}\right)=\mathrm{d}$, and the measuring error can be expressed as

$\eta\left(\mathrm{R}_{0}^{\prime \prime}, \mathrm{R}_{0}^{\prime}\right)=\frac{\mathrm{f}\left(\mathrm{R}_{0}^{\prime \prime}\right) \mathrm{R}_{0}^{\prime}-\mathrm{f}\left(\mathrm{R}_{0}^{\prime}\right) \mathrm{R}_{0}{ }_{0}}{\mathrm{f}\left(\mathrm{R}_{0}{ }_{0}\right)-\mathrm{f}\left(\mathrm{R}_{0}^{\prime}\right)}-\frac{\sigma\left(\mathrm{R}_{0}^{\prime \prime}\right) \mathrm{R}_{0}^{\prime}-\sigma\left(\mathrm{R}_{0}^{\prime}\right) \mathrm{R}_{0}{ }_{0}}{\sigma\left(\mathrm{R}_{0}{ }_{0}\right)-\sigma\left(\mathrm{R}_{0}{ }_{0}\right)}$

where $\mathrm{f}=\sigma$ when $\mathrm{R}_{0}<A$. Therefore, $\eta\left(\mathrm{R}_{0}^{\prime \prime}, \mathrm{R}_{0}^{\prime}\right)=0$ to random $A>R^{\prime \prime}{ }_{0}>R^{\prime}{ }_{0}$. When there is only one congestion link in the path, the path delay increase rate $\mathrm{K}$ measured is the delay increase rate of the tight link and $\mathrm{f}\left(\mathrm{R}_{0}\right)=\sigma\left(\mathrm{R}_{0}\right), \eta\left(\mathrm{R}^{\prime \prime}{ }_{0}, \mathrm{R}_{0}\right)=0$. There is no error in theory using formula (21) to get the path-available bandwidth. According to Theorems 2 and 3 , we know that $f\left(R_{0}\right)$ has a piecewise linear relation with $\mathrm{R}_{0}$, and the slope of $f\left(R_{0}\right)$ increases gradually with the increase of the congestion link caused by the increase of $\mathrm{R}_{0}$. According to the related approximate triangle theorem, it is easy to prove that the path-available bandwidth will be bigger than actual if it is calculated using $\mathrm{K}$ and corresponding $\mathrm{R}_{0}$ is measured when there are multiple congestion links.

\subsection{Identify the number of congested links in the path}

1) Relation Analysis between Path Delay Increase Rate and TimeRecognizing the number of congestion links is key to getting the correct available bandwidth.

The following analysis shows that the one-way delay increase $\mathrm{K}$ has a piecewise linear relation with the time $t$ under a fixed rate of the probing traffic when the buffer of the node in the path is limited.

We suppose that the congestion links are $L_{1}^{c}, L_{2}^{c} \ldots L_{u}^{c}$ and the rate of the data traffic beyond the bandwidth of one link $L_{i}^{c}$ is

$$
E s_{i}^{c}=R s_{i}^{c}+M s_{i}^{c}-C_{i}^{c}
$$


According to the law of "little," the occupied capacity of the buffer of the node is $O_{i-1}^{c}=M s_{i}^{c} *$ Delay $_{i-1}^{c}$ before sending the probing traffic. The buffer size of node $N_{i-1}^{c}$ on the sending end of $L_{i}^{c}$ is $B_{i-1}^{c}$ Delay ${ }_{i-1}^{c}$ is the sum of the queuing delay and the TX delay of node $N_{i-1}^{c}$, and the remaining buffer size is $B_{i-1}^{c}=B_{i-1}^{c}-O_{i-1}^{c}$. The overflow time of node $N_{i-1}^{c}$ is $t_{i}^{c}=\frac{B_{i-1}^{c}{ }^{\prime}}{E_{i}^{c}}$ It begins to lose part of the new package after a buffer overflow and the time interval between the time of the packages that are not be discarded entering the queue and the time of the packages sent is $\mathrm{d}_{i}^{c}=B_{i-1}^{c} / C_{i}^{c}$, which is constant obviously. The delay increase is zero at the moment, i.e., $\Delta \mathrm{d}_{i}^{c}=0$, and the delay increase rate of link $L_{i}^{c}$ is zero, $K_{i}^{c}=0$.

We sort the links $L_{1}^{c}, L_{2}^{c} \ldots L_{u}^{c}$ in ascending order according to the length of overflow time, and we get the links $L_{1}^{c}, L_{2}^{c} \ldots L_{u}^{c}$, whose corresponding overflow time and delay increase rate is $t_{1}^{t}, t_{2}^{t} \ldots t_{u}^{t}$ and $K_{1}^{t}, K_{2}^{t} \ldots K_{u}^{t}$.The path-delay increase rate $\mathrm{K}$ can be expressed as a function $\omega(\mathrm{t})$ about the time $\mathrm{t}$.

$$
\omega(\mathrm{t})=\left\{\begin{array}{l}
K, t \leq t_{1}^{t} \\
\sum_{i=1}^{u} \mathrm{H}_{i}^{c} \\
0, t>t_{u}^{t}
\end{array}, \text { when } L_{p}^{c}=L_{i}^{t}, \mathrm{i}<\mathrm{j}, t_{j-1}^{t}<\mathrm{t} \leq t_{j}^{t}, \text { exist }_{p}^{c}=0\right.
$$

In a particular case, $t_{1}^{t}=t_{i}^{t}=t_{u}^{t}, 1<\mathrm{i}<\mathrm{u}$, and

$$
\omega(\mathrm{t})=\left\{\begin{array}{l}
K, t \leq t_{1}^{t} \\
0, t>t_{1}^{t}
\end{array}\right.
$$

Therefore, $\omega(\mathrm{t})$ is a constant function about $\mathrm{t}$.

We can get the numbers of congestion links by getting the segments of the function $\omega(\mathrm{t})$ through identify technology of the segments of line segment.

2) Algorithm of Getting Segments of Straight LinesWe assume that the numerical sequence measured is $\omega\left(t_{1}\right), \omega\left(t_{2}\right) \ldots \omega\left(t_{N}\right)$, which we need pre-process. We accumulate the numerical sequence to get the numerical sequence $w\left(t_{1}\right), w\left(t_{2}\right) \ldots w\left(t_{N}\right)$ as data of the $y$ axis and make the time as the $\mathrm{x}$ axis. Then we can clearly watch out the tendency of the delay. The slope of a point in the linear fitting curve is the delay increase rate.

The key to recognizing whether there are multiple congestion links is to observe the segments of the linear fitting curve. If the segments are larger than two, there is more than one congestion link in the link.

Algorithm 1 Optimal partitioning algorithm of the set

The algorithm can be expressed as the best fitting of a set of data points and the segments $T$, i.e., the smallest sum of the distance between the data points and the fitting curve.

First of all, we make the difference of the data points in the set processed. We extract a point from each $\mathrm{N}$ point, and the point should satisfy the median of the $\mathrm{N}$ point, which can reduce the amount of computations to form a point set for the following steps. We divide the set of points in segments to get a non-empty subset sum and all partitions.

We calculate the imitative straight line of each subset and process the points in the subset with a linear least squares fit. The error of the subset sum is the sum of the squares of the distance between all points in the set and the imitative straight line. We calculate the error and record the quadratic sum and the variance of the error mean. In addition, we calculate the sum of errors in all sets to get the partition with smallest sum of errors.

The minimum variance, found from dividing the origin points into an m subsets sum, is expressed as $F_{m}(i, j)$, whose recursion formula is

$$
F_{m}(i, j)=\operatorname{Min}\left\{F_{1}(i, k)+F_{m-1}(k, j)\right\} .
$$

The time complexity of Algorithm 1 is $\mathrm{O}\left(\mathrm{N}^{4}\right)$ and the space complexity of the algorithm is $\mathrm{O}(\mathrm{N} * \mathrm{~N} * \mathrm{n})$.

Algorithm 2 Piecewise combination algorithm

The optimal partitioning set can be obtained from Algorithm 1, including $\mathrm{T}$ sub-collections

$$
\text { best_set }=\left\{\text { sque }_{1}, \text { sque }_{2}, \ldots \text { sque }_{T}\right\}
$$

The algorithm assumes that the error of the subset and the midpoint to the fitting straight line is in accordance with normal distribution, and it uses the hypothesis testing based on normal distribution to merge them.

According to the $3 \sigma$ principle of normal distribution, we can calculate the mean error of the point in set sque $i$ to the fitting straight line corresponding to set sque $_{i+1}$, with the fitting straight line corresponding to set sque ${ }_{i+1} \cdot \mu^{\prime}$.

If sque $i+1 . \mu^{\prime} \in$ [sque $_{i} . \mu-3 *$ sque $_{i} . \sigma$ sque $_{i} . \mu+3 *$ sque $\left._{i} . \sigma\right]$, when the confidence coefficient is $99.7 \%$, set sque ${ }_{i+1}$ is considered as a sample of the same normal distribution as set sque ${ }_{i}$, and set sque ${ }_{i+1}$ is combined with set sque . $_{\text {. }}$

Traverse best_set and combine to give a final number of subset $\mathrm{x}$. The time complexity of Algorithm 2 is $\mathrm{O}(\mathrm{n})$.

Based on the above analysis, when the input time of probing traffic is long enough, the value of $\mathrm{x}$ is at least 2 .

In the experiment, in order to enhance the recognition rate of Algorithm 2, the segments shorter than $1 / 10$ of the average length after combination are discarded.

Furthermore, the MultiLink-QuickAbw algorithm can even identify the overflow of the sending nodes on the congested links. Algorithm 3 decides whether to terminate the probing traffic.

Algorithm 3 Algorithm of judgment on the termination of probing traffic

The number of data packages we receive is $M$ at the receiving end, and we make the accumulated value of the delay increase and the time of arrival a point.

Running Algorithm 1 with these points as parameters and setting the segments $\mathrm{T}$ to 1 , we can get the linear fitting equation $\mathrm{y}=\mathrm{ax}+\mathrm{b}$, average error $\mu$, and the error variance $\sigma^{2}$. In addition, we calculate the average error $\mu^{\prime}$ between these points and the straight line $\mathrm{y}=\mathrm{b}$, and still test $\mu^{\prime}$ using the hypothesis of normal distribution. If , the delay increase rate of these packages is zero and the sending nodes of all congestion links are overflowed, which shows we should stop sending the probing traffic.

We require preprocessing before executing Algorithm 1. If the delay increase rate is zero, the number of points whose delay increase rate is zero should be extended to of the total numbers along the time axis ( $\mathrm{x}$ axis) to lower the error of Algorithm 1.

\subsection{MultiLink-QuickAbw Algorithm}

Algorithm 4We set the initial rate of probing traffic as . Loop, 
We send probing traffic and calculate the output rate when each receives $M$ probe packets at the receiving end. If is equal to , there is no congestion link. is the rate we need to increase. Then we set, GOTO NEXT.

If is not equal to, we execute Algorithm 3 with the data to judge whether we should send probing traffic or not. The sending time is . If we need to continue to send probing traffic, GOTO NEXT. After we stop sending probing traffic, we accumulate the relative delay of the receiving packages at the receiving end and get. We set the number of links in the network to plus one as the number of segments. Executing Algorithms 1 and 2, we can get $\mathrm{x}$, which is the number of segments. If $x$ is equal to 1 , the overflow time is too short. Then we set $M=M^{*} p,(1<\mathrm{p}<2)$,GOTO NEXT.

If $\mathrm{x}$ is bigger than 2 , there is more than one congestion link. Then we set, GOTO NEXT.

If $\mathrm{x}$ is equal to 2, there is only one congestion link. We can calculate the available bandwidth A with the delay increase rate K. If not assigned, we set GOTO NEXT. If is assigned, we set and . Substituting them into Equation 2-21, we can calculate the available bandwidth A, then we end the loop.

Next,

Sleep a length of , the length of must be long enough to make the buffer in the node congestion disappear. According to the law of "little," the limit of is . Because the available bandwidth is unknown, we would consider the congestion link as gone if we cannot receive the probing packages.

\section{GOTO LOOP}

END.

In the case where Algorithm 3 has achieved two K whose segment number is 2 , we can continue the iteration and widen the distance between the two $\mathrm{K}$ values, which is helpful for the accuracy of the calculation.

When Algorithm 3 is run second, in order to further decrease the probing time, the probing traffic rate can be set to the corresponding probing traffic rate when the last measured first number of segments is 2 . In practice, in order to reduce the error we can make several iterations and calculate the average of the available bandwidths. When QuickAbw algorithm first finds that there is only one congested link, record the number of packets before the buffer overflows as L. When the probing traffic is sent again, the number of packages should not exceed L. In this way, the measuring time and the network measurements are reduced.

\section{EXPIREMENT}

\subsection{Verify Probing Traffic Input-Output Curve Characteristics}

As shown in Figure 1, nodes N1, N2...N6 are connected by links L1, L2, ...L5. The capacity of these links are $\mathrm{C}(\mathrm{L} 1)=200 \mathrm{Mb} / \mathrm{s}, \mathrm{C}(\mathrm{L} 2)=155 \mathrm{Mb} / \mathrm{s}, \mathrm{C}(\mathrm{L} 3)=34 \mathrm{Mb} / \mathrm{s}, \mathrm{C}(\mathrm{L} 4)=10 \mathrm{Mb} / \mathrm{s}$, and $\mathrm{C}(\mathrm{L} 5)=200 \mathrm{Mb} / \mathrm{s}$, and the background traffic rates are :

. The corresponding available bandwidths are $A(L 1)=200 \mathrm{Mb} / \mathrm{s}$, $\mathrm{A}(\mathrm{L} 2)=3 \mathrm{Mb} / \mathrm{s}, \mathrm{A}(\mathrm{L} 3)=5 \mathrm{Mb} / \mathrm{s}, \mathrm{A}(\mathrm{L} 4)=3 \mathrm{Mb} / \mathrm{s}$, and $\mathrm{A}(\mathrm{L} 5)=200 \mathrm{Mb} / \mathrm{s}$. Sending probing traffic from N1 to N6, the rate rises from $0 \mathrm{Mb} / \mathrm{s}$ to $9 \mathrm{Mb} / \mathrm{s}$ with the step of $10 \mathrm{~Kb} / \mathrm{s}$. According to the simulation in MATLAB, we can get the relationship between delay increase rate $\mathrm{K}$ and .

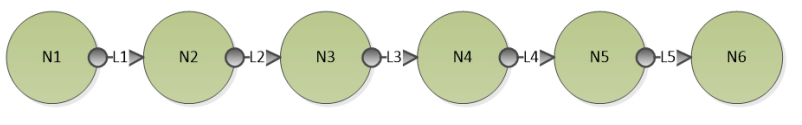

Figure 1: Network topology for experiment

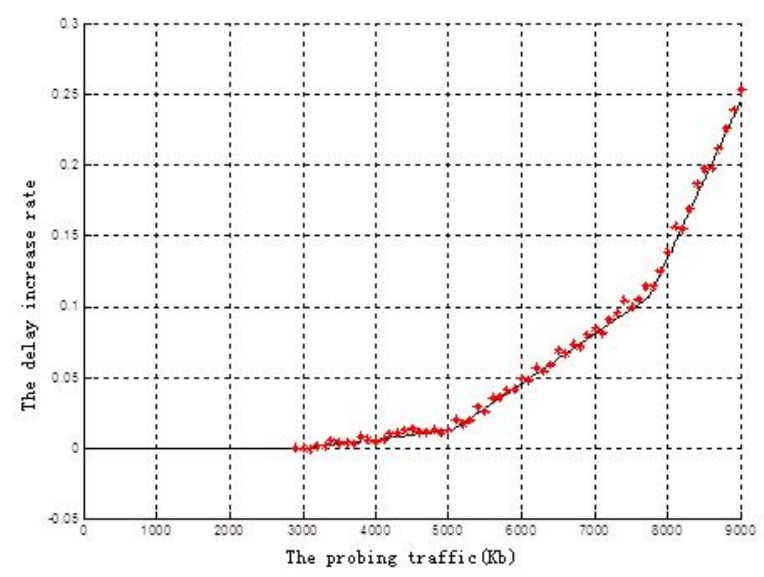

Figure 2: Delay rate theoretical curve (black line) and the corresponding simulation curve (red asterisk), when the probing traffic rate increased from 0 to $9000 \mathrm{~kb} / \mathrm{s}$

We get simulation data from the NS2 platform in this way: the same network environment is constructed in the NS2 environment, the probing traffic rises from $0 \mathrm{~KB} / \mathrm{s}$ up to $9000 \mathrm{~KB} / \mathrm{s}$, setting the step size to $100 \mathrm{~KB} / \mathrm{s}$. The size of the packet is set to 1000 bytes. We can only collect the delay increase rate before the probing traffic loses packet.

As can be seen in Figure 2 the first congestion link happens when the rate reaches $3000 \mathrm{~kb} / \mathrm{s}$, the second happens when $5050 \mathrm{~kb} / \mathrm{s}$, and the third at 7750 . From the results of the simulation we find that the curve acquired from NS2 fits the curve of the theoretical derivation.

\subsection{Path Delay Rate and Time Curve}

We use the network topology in Figure 1 and set $\mathrm{C}(\mathrm{L} 1)=200 \mathrm{Mb} / \mathrm{s}, \mathrm{C}(\mathrm{L} 2)=20 \mathrm{Mb} / \mathrm{s}, \mathrm{C}(\mathrm{L} 3)=100 \mathrm{Mb} / \mathrm{s}, \mathrm{C}(\mathrm{L} 4)=10 \mathrm{Mb} / \mathrm{s}$, and $\mathrm{C}(\mathrm{L} 5)=200 \mathrm{Mb} / \mathrm{s}$. We set the network background flow to , then the available bandwidth will be $\mathrm{A}(\mathrm{L} 1)=200 \mathrm{Mb} / \mathrm{s}, \mathrm{A}(\mathrm{L} 2)=7 \mathrm{Mb} / \mathrm{s}, \mathrm{A}(\mathrm{L} 3)=3 \mathrm{Mb} / \mathrm{s}, \mathrm{A}(\mathrm{L} 4)=9 \mathrm{Mb} / \mathrm{s}$, and $\mathrm{A}(\mathrm{L} 5)=200 \mathrm{Mb} / \mathrm{s}$.

We set the probing traffic rate at $8 \mathrm{Mb} / \mathrm{s}$, the number of packets as 700 , and the size as 1000 bytes. The measured results are shown in Figure 3, where the $x$-axis represents the time and the $y$-axis the time delay. We can see from the figure that the algorithm detects flow changes at the receiving end, but the change trend of the delay cannot be observed.

We can observe the trend of detect flow delay by cumulating the delay variation. Make Arithmetic 2.1 and Arithmetic 2.2 piecewise fitting, and set the maximum number of segments to 5 (number of link), as shown in Figure 4 


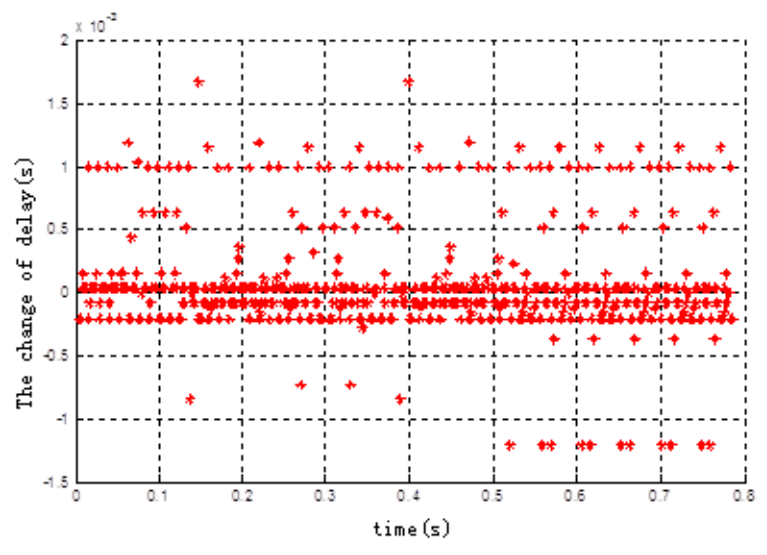

Figure 3: Corresponding variation of packet delay, when the injection rate is $8 \mathrm{Mb} / \mathrm{s}$ and te number of pack is 700 , measuring sustained $0.8 \mathrm{~S}$

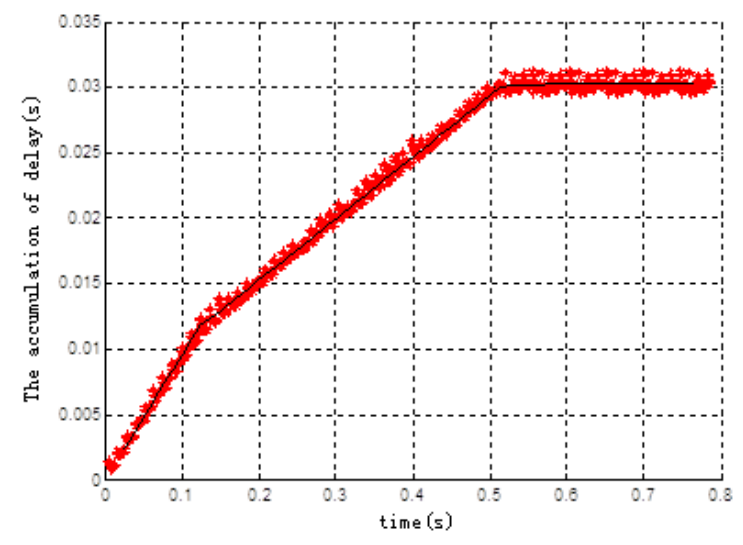

Figure 4: Curve fitting after segmentation

The curve consisting of black points is the result after we adopt Arithmetics 1 and 2 with piecewise fitting. Algorithm 2 finds the section number is 3 , which indicates there are two congestion links. The packet loss rate of the curve in the time interval is shown in Figure 5

The piecewise linear curve fit is shown in Figure 4, and the slope of each section shows the time delay corresponding to the growth rate. In Figures 4 and 5, we can see packet loss, and the delay rate decreases in 0.13 seconds with the congestion link sending node overflows. In 0.52 seconds all the congestion link send node overflows, delay growth rate to zero, the number of link congestion occurred to 2, Algorithm 1 and Algorithm 2 identify the number of curve segment is 3 .

When the detecting flow injection rate is $5 \mathrm{MB} / \mathrm{s}$, the number of package for 700 , the delay variation at the receiving ends is as shown in Figure 6

Accumulating the delay changes, the corresponding delay change curve is as shown in Figure 7

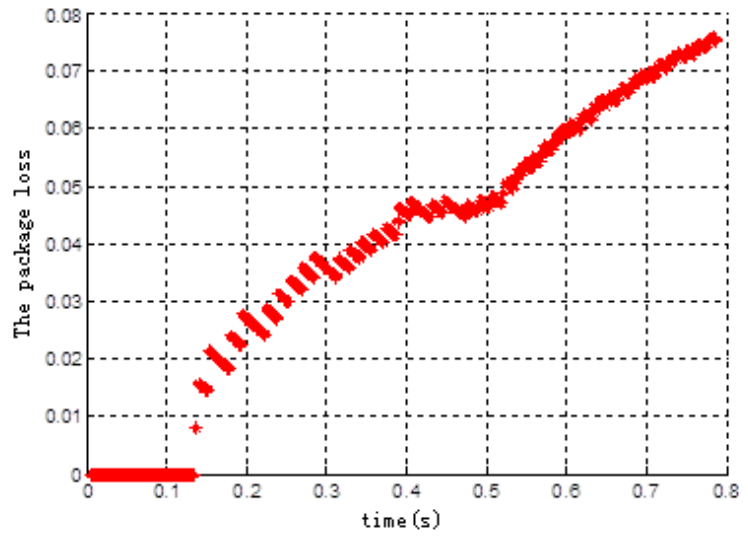

Figure 5: Packet loss rate curve when the probing traffic is $8 \mathrm{Mb} / \mathrm{s}$

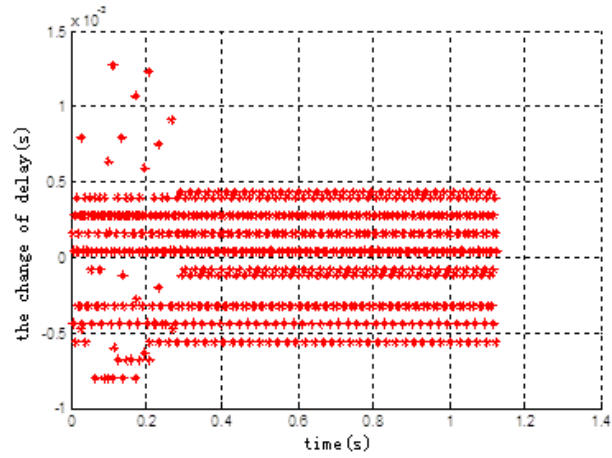

Figure 6: Correspond variation of packet delay, when the injection rate is $5 \mathrm{Mb} / \mathrm{s}$ and the number of pack is 700 , measuring sustained $1.12 \mathrm{~S}$

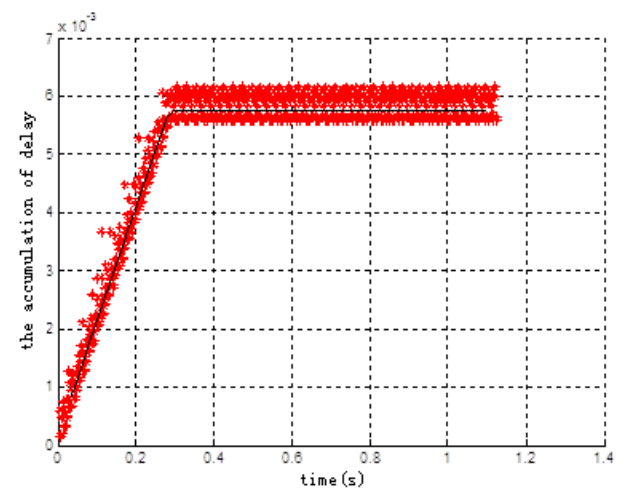

Figure 7: Curve fitting after segmentation when the probing traffic is $5 \mathrm{Mb} / \mathrm{s}$ 


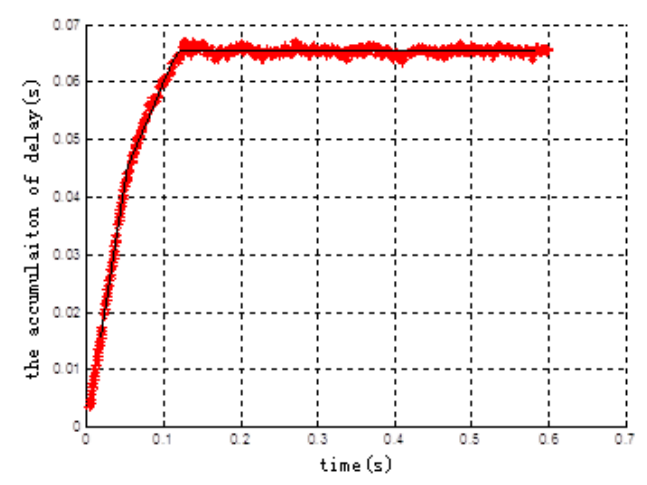

Figure 8: Corresponding cumulative curve delay variation when probing traffic rate is $60 \mathrm{Mb} / \mathrm{s}$

Figure 7 shows that the link congestion link exists in one place, and Algorithms 1 and 2 to identify the number of curve segments as 2 . Algorithms 1 and 2 under these two input rates more accurately identify the number of piecewise curves, but in some cases the cumulative delay change curve segment number is not easy to identify, such as detection of flow rate is $60 \mathrm{MB} / \mathrm{s}$, its cumulative delay change curve as shown in Figure 8

When the detecting flow injection rate reaches $60 \mathrm{MB} / \mathrm{s}$, the corresponding cumulative delay change curve slope of change is not obvious, and after the delay rate reaches 0 the jitter is larger; in $0.052 \mathrm{~s}, 0.078 \mathrm{~s}$, and $0.12 \mathrm{~s}$ are sending node spilling. In this case it is difficult for Algorithms 1 and 2.2 to identify the path of the congestion link number: $0.078 \mathrm{~s}$ overflow in the test case failed to recognize and identify the congestion link number as 2 , and the actual congestion link number was 3 . But as long as it can correctly identify that the congestion link is greater than 1 it will not affect the iterations of the algorithm.

\subsection{Measuring Error of Validation under Numbers of Congestion Links}

The network structure is constructed in the same way as done in III.A, and the initial rate is set to $15 \mathrm{MB} / \mathrm{s}$. We can get the acquisition delay rate and the corresponding rate, therefore, there are two congestion links, at the initial iteration to obtain and corresponding sending rate to calculate the available bandwidth. Figure 9 shows different transmission speeds of the available bandwidth.

Starting the iteration with $15 \mathrm{M} / \mathrm{s}$ at the sending end until the sending rate reaches $3972.98 \mathrm{~Kb} / \mathrm{s}$, we find that it has conducted $50 \mathrm{sec}-$ onds and 74 times. We calculate the available bandwidth using the rate of sending end as and the corresponding delay increase rate as

The results show that the available bandwidth measured is larger than the actual bandwidth available, despite the fact that there is only one congestion link.

Figure 9 shows that, in the two data collected for calculating the available bandwidth, as long as there exists more than one congested link when collecting data, the measured result is larger than the actual value. This is consistent with the derivation of Section II.B.

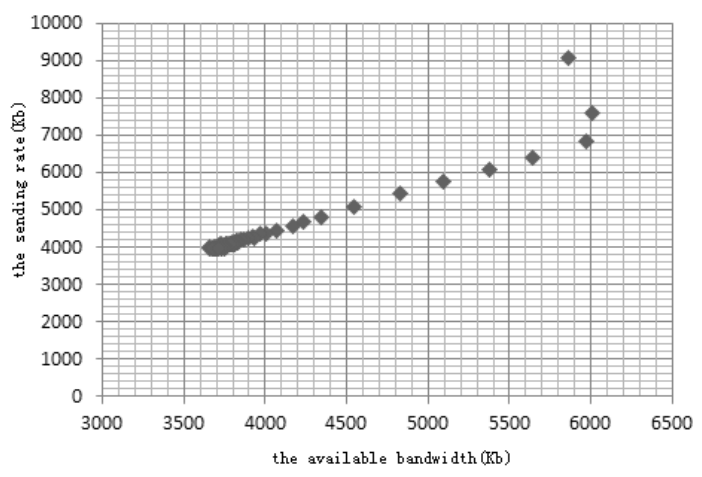

Figure 9: Measurement error when there are two congested links

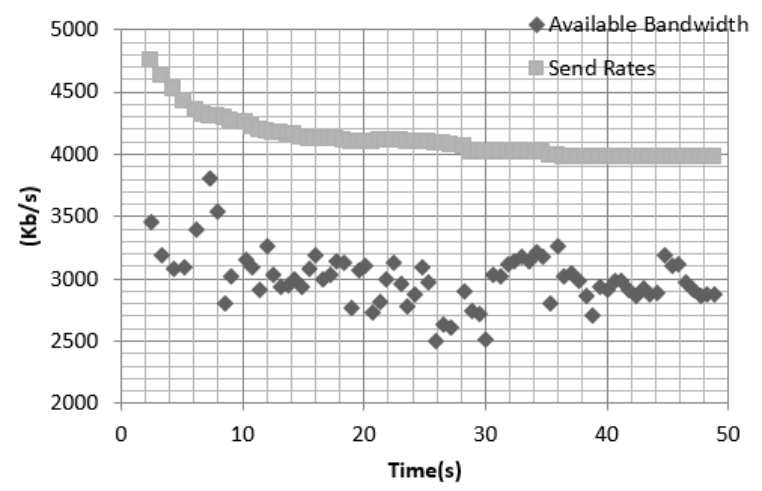

Figure 10: Cure of available bandwidth when the initial rate is $7 \mathrm{Mb} / \mathrm{s}$

\subsection{Measuring Example of CBR Background Traffic}

Set the background traffic is CBR, sending rate is constant, and the packet size is $1500 \mathrm{~B}$. $\mathrm{M}$ in Arithmetic 2.3 is set to 100 , which means each received 100 package judged whether the entire sending node congested links are overflow. We consider that the probe packets disappear from a path after $0.2 \mathrm{~s}$ when the last probe packet has been received at the receiving end. The probing package size is set to $1000 \mathrm{~B}$. The initial rate is set to $20 \mathrm{MB} / \mathrm{s}$. Each iterations until all nodes overflow. The measurement process is shown in Table 1 In Table 1, seven iterations took 5.5 seconds. Using the fifth iteration rate for, the corresponding delay growth as, the sixth iterative calculation of the available bandwidth is $2.7527 \mathrm{Mb} / \mathrm{s}$. From the seventh time using the correspond data we know that the available bandwidth is $3.04872 \mathrm{Mb} / \mathrm{s}$, which has a very small error from the actual value.

\subsection{Factors that Affect Measuring Precision}

We use the same environment as the previous section, starting the iteration at $7 \mathrm{Mb} / \mathrm{s}$ to obtain the corresponding data and , and then calculate the available bandwidth. Figure 10 shows the calculation results. 
Table 1: Measurement process under initial probing rate $20 \mathrm{Mb} / \mathrm{s}$

\begin{tabular}{lllll}
\hline \#Iterations & Sending rate $(\mathrm{Mb} / \mathrm{s})$ & Receiving rate $(\mathrm{Mb} / \mathrm{s})$ & Number of congestion links & Measured results $($ Mb/s) \\
\hline 1 & 20.00 & 9.30 & 3 & - \\
2 & 9.30 & 7.90 & 2 & - \\
3 & 7.90 & 7.23 & 2 & - \\
4 & 7.23 & 6.92 & 2 & - \\
5 & 6.92 & 1 & - & 2.75 \\
6 & 6.76 & 1 & 3.04 \\
7 & 6.51 & 6.51 & 1 & \\
\hline
\end{tabular}

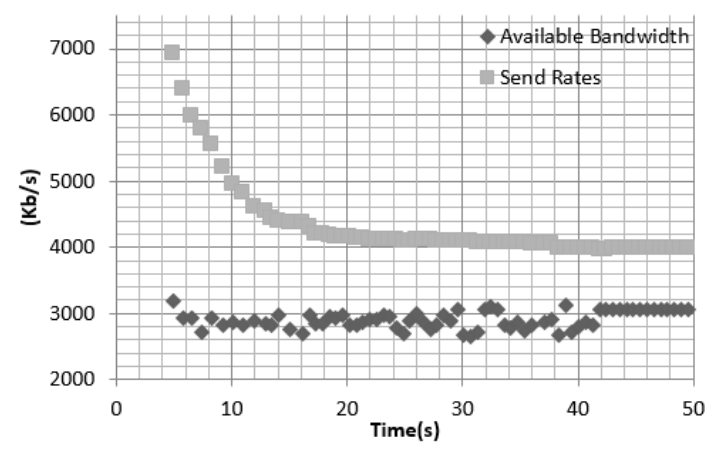

Figure 11: Cure of available bandwidth when the initial rate is $5 \mathrm{Mb} / \mathrm{s}$

From Figure 10 it can be seen that, along with the iteration, the sending end rate reduces more and more slowly, and eventually stops $(3975.35 \mathrm{~Kb} / \mathrm{s})$. At this time, the available bandwidth calculated is no longer change at $3069.14(\mathrm{~Kb} / \mathrm{s})$. From Figure 3-10 we cannot conclude that the bigger the rate difference of the two probing traffics, the more accurate the result is. Before the iteration speed is constant, the available bandwidth measured and the difference between the two detecting flow rates has no significant regularity. The average result is $2910.26 \mathrm{~Kb} / \mathrm{s}$, lower than the actual value, and the variance is 18746 . In the measurement, the maximum error is $404.21 \mathrm{~Kb} / \mathrm{s}$. Due to the fact that the gap between two probing traffics rate is at least $537 \mathrm{~Kb} / \mathrm{s}$, we need further experiments for when the probing traffic rate gap is small. Therefore, we set the initial rate at $5 \mathrm{Mb} / \mathrm{s}$ and $4.3 \mathrm{Mb} / \mathrm{s}$, and the corresponding measurement curves of available bandwidth are as shown as Figures 11 and 12

Figure 11 Shows that when the initial rate of the probing package is $5.0 \mathrm{Mb} / \mathrm{s}$, variance of the measured results increases obviously. When the difference between the two probing packages is $700 \mathrm{~KB} / \mathrm{s}$ or less, the measurements jitter seriously and the error is large.

Figure 12 shows that the sending rate in the sender become less, when the initial rate is $4.3 \mathrm{Mb} / \mathrm{s}$. The low initial rate affects the accuracy of the results because it will lead to small differences between two measurements. In the above experiment, the average of multiple measurements is very close to the real available bandwidth; therefore, it can be measured by taking the average of multiple iterations to achieve higher accuracy.

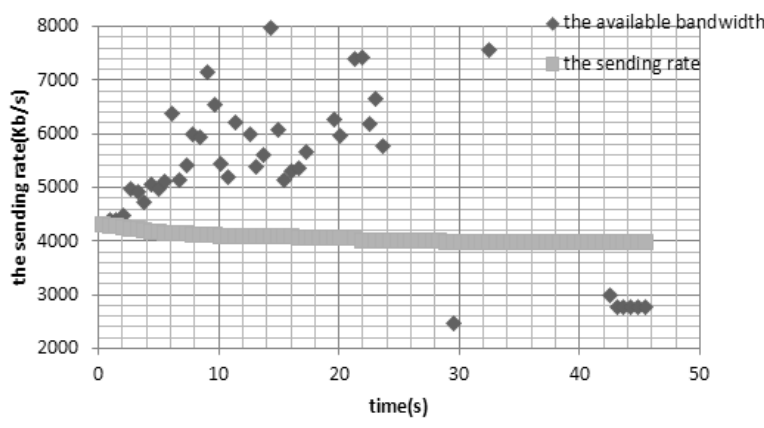

Figure 12: Cure of available bandwidth when the initial rate is $4.3 \mathrm{Mb} / \mathrm{s}$

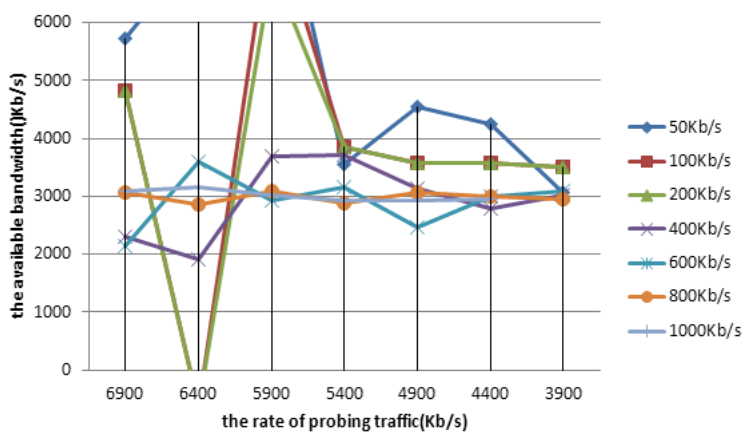

Figure 13: Measurements at different intervals to detect the flow rate and the flow rate of the first probe traffic

To further analyze the influence of rate interval of the probing traffic and rate on the accuracy, we set the rate interval of the probing traffic to $50 \mathrm{~Kb} / \mathrm{s}, 100 \mathrm{~Kb} / \mathrm{s}, 200 \mathrm{~Kb} / \mathrm{s}, 400 \mathrm{~Kb} / \mathrm{s}, 600 \mathrm{~Kb} / \mathrm{s}, 800 \mathrm{~Kb} / \mathrm{s}$, $1200 \mathrm{~Kb} / \mathrm{s}$, and $1600 \mathrm{~Kb} / \mathrm{s}$, and the calculation interval as $500 \mathrm{~Kb} / \mathrm{s}$ from $6.9 \mathrm{Mb} / \mathrm{s}$ to $3.9 \mathrm{Mb} / \mathrm{s}$. The results are shown in Figure 13

Figure 13 shows that the rate difference is small $(50 \mathrm{~Kb} / \mathrm{s}, 100 \mathrm{~Kb} / \mathrm{s})$, the measurement error is large, and the jitter serious, especially with the high initial rate. Initial probing traffic rate, the closer it gets to the available bandwidth, the greater the difference between the two probing traffic rates, calculate the more accurately available bandwidth. When the difference between the probing traffic rates increased, the high initial probing traffic rate had a reduced impact on the measurement results. But in the actual cases, the lower initial 
rate will make it smaller that the sending each iterative reduced (as shown in Figure 12), so it is not conducive to achieve result accurately.

\section{RELATED WORK}

Available bandwidth measurement can be divided into active measurement and passive measurement. Passive measurement is less invasive and has little impact on the network, but due to environmental constraints, active measurement methods are often required. Active measurement typically sends probe packets from the sending node to the receiving node, and then we can get delay variation and packet loss rate of the packet in the receiving node to calculate the available bandwidth. Active measurement can be divided into probe gap model (PGM) and probe rate model (PRM). The available bandwidth measurement tools IGI [2] and Spruce [3] using the PGM model estimate the available bandwidth by using the input and output interval dispersion of the probe packet pairs. PRM mode which includes measurement tools Pathload [4], Pathchirp [5], Yaz [6], Assalo [7], Topp [8], etc., calculates available bandwidth by calculating the trend of one-way delay change of detected packets. These active measurement algorithms have a common feature that they need to adjust the transmission rate of the probe packet at the sending end until it is equal to the transmission rate at the receiving end, which eventually leads to a long measurement time. To achieve high accuracy, the PGM model algorithm uses multiple repeated measurements to calculate the mean value. Due to the long measurement time, PRM model algorithm will pour a large number of detection messages into the network, affecting the normal work of the network.

In recent years, with the rapid growth of network bandwidth and the popularization of $5 \mathrm{G}$ network, the research on the measurement of available bandwidth is still continuing. For example, [9] [10] [11] [12] involves the study of available bandwidth. Wireless networks. Minprobe [13] uses a low-cost intermediate box to measure the available bandwidth on a $10 \mathrm{Ge}$ network. [14] proposes an ABW estimation method with less overhead than existing methods. The above classification method is suitable for most new measurement methods.

With the emergence of these new methods, some studies such as [15] [16] [17] have focused on the evaluation of ABW tools. These studies try to find the key factors that affect the measurement results of available bandwidth, such as network load conditions and the characteristics of the injected probe packet. The study in literature [17] shows that the measurement accuracy of available bandwidth is proportional to the detection volume. While improving the measurement accuracy, it is difficult to reduce the number of detection packets sent.

In our previous work[1],we implemented the QuickAbw method. Both simulations and contrast test in real network environment show that QuickAbw has higher measurement accuracy and shorter measurement time than other compared tools in a network with single bottleneck-link under a variety of common background traffic, it outperforms comparison tools under most network loads, however, QuickAbw is only suitable for single congested links in the path.

\section{CONCLUSIONS}

This research further expands our previous research, enabling QuickAbw to measure bandwidth under a more complex MultiCongestion-Links path. In addition to measuring the large amount available, the algorithm can also be used to measure the number of congested links. In the experiment, we analyzed the key factors that affect the measurement error. In actual online applications, these factors can be referenced to set appropriate values to obtain a compromise between measurement accuracy, measurement speed, and network intrusion.

\section{ACKNOWLEDGMENTS}

This work is supported by The National Key Research and Development Program of China (No.2020YFB1006100, No.2020YFE0200500 and No.2018YFB1800200) and Key research and Development Program for Guangdong Province under grant No. 2019B010137003.

\section{REFERENCES}

[1] Shi, Junzheng, et al. "An Quick Available-Bandwidth Measurement Method Based on Link Delay Growth Rate." Proceedings of the 2020 8th International Conference on Communications and Broadband Networking. 2020.

[2] $\mathrm{Hu}, \mathrm{N}$. and Steenkiste, P., 2003. Evaluation and characterization of available bandwidth probing techniques. IEEE journal on Selected Areas in Communications, 21(6), pp.879-894.

[3] Strauss, J., Katabi, D., Kaashoek, F. and Prabhakar, B., 2003, March. Spruce: A lightweight end-to-end tool for measuring available bandwidth. In Proc. of the Internet Measurement Conference (IMC).

[4] Jain, M. and Dovrolis, C., 2002. Pathload: A measurement tool for end-to-end available bandwidth. In In Proceedings of Passive and Active Measurements (PAM) Workshop.

[5] Ribeiro, V.J., Riedi, R.H., Baraniuk, R.G., Navratil, J. and Cottrell, L., 2003, April. pathchirp: Efficient available bandwidth estimation for network paths. In Passive and active measurement workshop.

[6] Sommers, J., Barford, P. and Willinger, W., 2006, June. A proposed framework for calibration of available bandwidth estimation tools. In 11th IEEE Symposium on Computers and Communications (ISCC'06) (pp. 709-718). IEEE.

[7] Goldoni, E., Rossi, G. and Torelli, A., 2009. Assolo: An efficient tool for active end-to-end available bandwidth estimation. International Journal on Advances in Systems and Measurements, 2(4), pp.283-292.

[8] Melander, B., Bjorkman, M. and Gunningberg, P., 2000, November. A new endto-end probing and analysis method for estimating bandwidth bottlenecks. In Globecom'00-IEEE. Global Telecommunications Conference. Conference Record (Cat. No. 00CH37137) (Vol. 1, pp. 415-420). IEEE

[9] Paul, A.K., Tachibana, A. and Hasegawa, T., 2016. An enhanced available bandwidth estimation technique for an end-to-end network path. IEEE Transactions on Network and Service Management, 13(4), pp.768-781.

[10] Li, M., Claypool, M. and Kinicki, R., 2008, October. WBest: A bandwidth estimation tool for IEEE 802.11 wireless networks. In 2008 33rd IEEE Conference on Local Computer Networks (LCN) (pp. 374-381). IEEE.

[11] Dely, P., Kassler, A., Chow, L., Bambos, N., Bayer, N., Einsiedler, H. and Peylo, C., 2014. BEST-AP: Non-intrusive estimation of available bandwidth and its application for dynamic access point selection. Computer Communications, 39, pp.78-91.

[12] Castellanos, W., Guerri, J.C. and Arce, P., 2019. Available Bandwidth Estimation for Adaptive Video Streaming in Mobile Ad Hoc. International Journal of Wireless Information Networks, 26(3), pp.218-229.

[13] Wang, H., Lee, K.S., Li, E., Lim, C.L., Tang, A. and Weatherspoon, H., 2014, November. Timing is everything: Accurate, minimum overhead, available bandwidth estimation in high-speed wired networks. In Proceedings of the 2014 Conference on Internet Measurement Conference (pp. 407-420).

[14] Matsuda, T. and Ishihara, S., 2020. Bandwidth Control Method and Available Bandwidth Estimation Method for Aggregated Traffic. Journal of Information Processing, 28, pp.31-43.

[15] Guerrero, C.D., Salcedo, D. and Lamos, H., 2013. A clustering approach to reduce the available bandwidth estimation error. IEEE Latin America Transactions, 11(3), pp.927-932.

[16] Salcedo, D., D Guerrero, C. and Martinez, R., 2018. Available bandwidth estimation tools metrics, approaches and performance.

[17] Morillo, S., David, D., Guerrero, J. and Guerrero, C.D., 2017. Overhead in available bandwidth estimation tools: Evaluation and analysis. 